\title{
The Phenomenon of Migration in the Light of the Systems Theory
}

\author{
Prof. Ludmila Gegel
}

Russian State Technological University, Moscow, Russia

Prof. Giulia Frolova

Astrakhan State University, Astrakhan, Russia

\section{Doi:10.5901/ajis.2013.v2n11p112}

\section{Abstract}

\begin{abstract}
Modern society is a self-organizing system. When resources (population) start to decrease, the system opens for the influx of foreign elements, hoping to adapt them. Migration is an example of this process. However, new items are not always able to assimilate. Sometimes they are so different that they are not compatible with the host system. Such "aliens" either quietly parasitize on the body of a recipient, or organize their own micro-systems, sprawling like a cancer and destroy the algorithm of the host system. A resource-scarce system is able to survive only by choosing items which are more or less similar in nature and then the system redistributes, splits and disposes them in itself. New elements will fuel the system, but in a different way than relative elements. This will change the system, its borders will evolve (mutate) and change. So the migration processes lead to the transformation of language, culture and behavior. As a result, transnational communities are formed. The traditional concept of nation (country) ceases to exist. The role of boundaries is transformed and is reduced to a nominal marking of a territory. Border areas are the bright example of it now. Governments are trying to apply the policy of integration, granting citizenship for migrants, but it is not always successful. The "new" citizens do not share the dominant culture. In opposite, they actively broadcast their way of life in a new territory. Previously, there was an identity between citizenship and belonging to the nation, but nowadays the situation has changed. Cultural and ethnic unity ceases to be an essential characteristic of a nation. These processes are still in the early stages, but they are becoming a world trend. It also Influenced Russia. Forthe last 10 years in Russia "human resources" crisis has been progressing. In 2025 Russia, like many European countries, will feel all the features of the system resource crisis. Searching for ways to resolve it today - is a strategic task, a question of survival.
\end{abstract}

Social transformations of XX-XXI centuries led to understanding that psychologically a man adapts to changing environment conditions slowly than from physical standpoint. To survive in an unstable social environment, one should search for successful models of social and economic behavior, adapted to a new reality. This process became agents for large-scale migration accelerated marginalization of population and the polarization of society in material and sociocultural terms. In this regard, the consideration of the social structure and system changes in regions with high polyethnicity and migration activity is of current interest.

The other aspects of relevance are:

- The importance of the development of methods of a system meta-approach to the analysis of social transformations of modern societies. Also the use of general principals of general systems theory (GST) gives insight into cause-and-effect relationship between processes and lets you find out the interdependence of the features of the social space (region), the mentality of its inhabitants and their overall outlook;

- A demand for system analysis of the migration situation in the world, the study of its features in specific regions and the impact on various areas of public relations, as well as the direction and prospects of interaction of migrants and recipient population of polyethnic areas.

Our research is based on the synthesis of systemic and sociological approaches, which in its turn defines the logic of presentation.

Focusing on the principles of general theory of complex dynamical systems enables to avoid the narrowness of methodological paradigms. It extends the heuristic potential of humanities studying the societies which are in the phase of interstadial civilization transition, civilization breaks and other unstable states.

Synthesis of the main ideas of GST and a socio-cultural approach allows to understand a society as a selforganizing system, adaptable to resource potential of the medium. Limited resources motivate the system to the search of a new mode (modality) of existence which meets demands of a basic system algorithm. The algorithm is a consistent 
mode of action (thinking), system elements (individuals, groups) that provides behavior necessary for survival. It is reflected in a value scale and limits deviations from an optimal (for the system) activity stereotype.

The algorithm is given by external and internal factors: a type of medium interaction (natural resource factor) and a type of intrasystem relation (organizational factor).A subsystem controls a basic algorithm observance in two forms: hierarchical and pluralistic. As for the first form, all the subsystems are subject to the center and differentiated by the degree of access to resources and security (e.g. Eastern societies). There is no center-dominator in the polysystem; all the elements are equally admitted to resources consumption and secured (e.g. European societies). Both system forms of existence are reasonable and valuable. Ideology of individualism is formed in pluralistic systems, as a unit of this system is a self-motivated individual. Collective world perception prevails in hierarchical systems, as hierarchy is possible only in a group. Sociocultural systems are implemented by local civilization modality. Exhausting resources of one modality, the system goes into a dynamic mode (new modality) which corresponds to the demands of its main life activity algorithm at a new level. In different civilization modalities of the system, its value priorities can change, but on the whole they are to correspond to a general scale, given by a system algorithm. It explains the stability of mentality of system elements (residents). Changing a form of management in any system and transferring it to a different type of civilization modality (e.g. due to migration overpressure on the part of representatives of a different sociocultural matrix), not corresponding to the algorithm demands, leads to the system collapse. Thus, synthesis of system and sociocultural ideas enriches our research, revealing the essence of nonlinear processes in complex social systems, genic causality of a form of control in any type of a system and the importance of its value-cultural core. In this case, metasystem trends of global civilization cycles are taken into account. World civilization (metasystem) implies the balance of systems of various types.

The search of new civilization modes by several systems of different type means a phase transition of the metasystem to the next evolutionary stage. These "changes of epochs" are periods of civilization breaks and cultural clashes, accompanied by deformation of the system algorithms, destruction of value-normative models, sustained reformations, migratory waves and society chaotization. In such periods, "duality" between usual and new values from different sociocultural systems, gradually puts people in ressentiment ("sociocultural nihilism" "self-poisoning of the soul" - M. Scheler). Ressentiment, denying a value system, makes social self-sentiment accessible only through destructive acts. This fact explains destructive behavior of migrants in a new society or xenophobia of the recipient population, which is in the minority. Marginalized world perception is formed in a prolonged state of ressentiment.

In the period of ressentiment, values are substituted by so-called "philosophy of mud", destruction. Under the circumstances, ethnicity and/or religion, minimally connected with social dynamics, becomes for migrants the only one value or stratification criterion.

Extrusive factors can be: civil riots, disorders connected with threat or use of force; prosecution on political, national, religious or other reasons; changes in the political situation and the political system, putting individuals or social groups in a vulnerable situation, environmental and natural disasters, economic stagnation, the implementation of some government programs connected with the resettlement of people against their wishes. Personal responses to external threats can often be a decision to move (away of risks, to more stable places, where it is possible to implement usual algorithms) which is the root cause of migration waves of modernity.

The subject of internal and external migration is also relevant to Russia, especially its southern and eastern territories. Migratory waves create challenges to regional and national security. To solve this problem, it is required to form a suitable migration policy. We cannot be isolated from migrants (as an isolated system is not viable).Indeed, we need them, because it is an exchange of resources with external medium. However it should be profitable both for the system-recipient and elements of external medium (migrants). It should not be just a controlled process. You should monitor in accordance with a basic algorithm of the system life, with demands of the modality in which the system exists. In our research, migrants are considered to be people, whose realization of vital strategies is possible only providing a certain transformation and a receiving society. You can trace the analogy with biological systems. A migrant is not just a passive, nutrient element, absorbed by the system. He is an active subject, invading the social system (like a bacteria or a virus cell in the organism). He can co-exist together with the system on mutually beneficial terms, can parasitize on it, and can destroy it. Variants of invasion depend on protection mechanisms and the system adaptation (its immunity). Unfortunately, there is a state crisis in the sphere of regulation of migration flows, which is evident from the lack of a balanced immigration policy. At the same time, one of the signs of a successful immigration policy is an integration of migrants suitable to the needs of a host system to a local society; i.e. absorption of new resource elements with the maximum mutual benefit.

One of the key problems here is to avoid/minimize possibilities of formation of migration enclave with their criminal, sociocultural, political threats to the national security. In turn, an indicator of social security is correspondence of 
standards of living to the world standards; increasing of regional property and financial sources to ensure social policy; effective using of region resources, working places, scientific and educational level of population, infrastructure, political stability and ethnic balance.

Modern migration processes take place against the backdrop of globalization.

In the mid-XX century, migration was large-scaled, responding to fluctuating impulse of a social metasystem (phase transition). Different local social systems began a difficult process of transition to a more difficult and higher level of existence (modality).This process is associated with the collapse of the usual forms of life, which, of course, scares people. At the beginning of the article we mentioned that psychologically a man adapts to changing environment conditions slowly than from physical standpoint. The system diffusion, its resources deprives a man of a familiar worldview. He tries to escape, hide from changes in zones less affected by system transformations. Occasionally, these zones are the core of metasystem (developed countries, which realize basic algorithm of a given modality). However it is impossible to stop evolution, developed countries are also involved in the transformation process. Being in a transitive state, they become more vulnerable to external factors - migration invasions. In such a difficult situation even the most reasonable decision concerning mass population movement causes fear over migration- fear of being in a trap. Any form of migration is believed to be a negative force, destroying a society and hindering effective management. Because of it, ultra-radical ideas and slogans take place. As a result, migration is political in nature and begins to affect state and national structures, becomes a catalyst of transformation of a political matrix and a donor country and a recipient country.

However it is important to understand that migration is an inevitable process, a characteristic of the epoch of a transformation global social system. It makes no sense to treat it as a sociocultural evil or as a panacea for depopulation in a region.

Migration is an intrinsic and inevitable process which is gaining strength. It is necessary to organize and correct it in accordance with modal needs of the system.

Taking into account diversity and different directions of migration flows, volume of moving population, it is possible to say that nowadays migration processes have exceeded the limits of a self-organizing system and their spontaneous development is dangerous. For countries of origin, migration can be a factorl obstacle for their future development (brain drain, loss of young and active work force, reduction of population reproduction).Positive sides of these processes are sending young unemployed people outside excess working regions, as they can be a source of a social strain; the use of returnee's experience.

For recipient countries, large scale human interventions have both positive and negative sides. Positive sides are to fill empty niches in the sectors of little prestige and in the sectors of high technologies; a negative one is a chance to get ethnic/religious trouble zones on your territory. Domination of the first or the second variants can depend on various reasons, first of all it is a migration nature and the second one is a government activity.

Migration processes lead to complication and transformation of social and political institutions, a language, culture, models of behavior both in host countries and countries of origin. As a result, the spread of transnational communities is accelerating, that is often considered to be a challenge to a traditional understanding of a nation; i.e. a concept of a nation (a country, a state) ceases to be filled with a traditional content, namely, the unity of the society, a titular ethnic group, economy, civil institutions, the monetary system, army, language, culture in unified national borders. A role of borders is transformed and comes to a formal marking of a national territory. In other words, national borders separate one national unity from others in a less degree. Democratic states implement a policy of migrant integration by conferment of nationality. However, a policy of formal assimilation often fails and ethnic communities outside their country develop and grow. These "new citizens" do not become a part of a nation in a traditional sense, i.e. do not share its culture, its mode of life. These processes are observed in the initial stage, but they become a part of the world trend. It is probably because of new elements in a new area enjoy all the benefits of a system-recipient and continue to act, using their own algorithm (Muslim diaspora, Chinese communities etc.) A new, relatively comfortable reality does not motivate them to energy consumption, connected with the change of the usual sociocultural algorithm. If there is no algorithm, there is no assimilation in a new system. Migration policy of a host country should consider these processes and build up relationship from the outside so that to make them change their system algorithm. It must be unfavorable for a migrant to stay in a new matrix, ignoring its system requirements (education, qualification, language, social programs, tax policy, a type of document flow, limitation of civil rights and others tools of correction of a system algorithm)

In our opinion, blurring of state boundaries, its national structures, institutions will occur due to a latent and evident activity of migration processes. All these mentioned things raise the issue of migration management to the level of a high priority task facing all the states. 


\section{References}

Gegel L.A. Frolova Y.S. Education and regional youth values. //Higher education in Russia. - 2007. - N 7.

Vallerstein I The end of a familiar world. - Moscow. Logos Press. -2003.

Dmitriev A.V. Migration: conflict dimension. - Moscow. Alfa Press. - 2012.

Milovanov V.P. Unbalanced social and economic systems: synergetic and self-organization. - Moscow. URS. - 2011.

Panarin A.S. Strategic instability in XXI century. - Moscow. Algorithm. - 2007.

Frolova Y.S. The influence of migration flows on the development of a modern South Russian polyethnic region. - Balashikha. MTU. 2013.

Frolova Y.S. A phenomenological analysis of value orientations of a transitive society. - Moscow. MOSU. -2001.

Udina T.N. Sociology of migration : to a formation of a new scientific direction. - Moscow. Academic Project. - 2010. 